\title{
A SIMPLER PROOF THAT COMPACT METRIC SPACES ARE SUPERCOMPACT
}

CHARLES F. MILLS

\begin{abstract}
AbSTRACr. We give a simpler proof that every compact metric space is supercompact.
\end{abstract}

In 1969 De Groot [5] introduced the notion of supercompactness (see definition below). All supercompact spaces are compact by the Alexander subbase lemma; the converse of this was recently shown false by Bell [1].

This leaves the question of what hypotheses on a space, beyond compactness, enable one to conclude that it is supercompact. Strok and Szymański [7] have shown, in particular, that all compact metric spaces are supercompact; their proof, however, is very complicated.

The purpose of this note is to offer a simpler proof. We have been advised that our proof is similar to one of van Douwen [4], though ours is somewhat simpler.

The author has recently shown [6] that all compact groups are supercompact.

A collection $\mathcal{L}$ is said to be linked if whenever $A, B \in \mathcal{L}, A \cap B \neq \varnothing$. $\mathscr{B}$ is binary if for every linked $\mathcal{C} \subset \mathscr{B}, \cap \mathcal{L} \neq \varnothing$. A space $X$ is said to be supercompact if $X$ has a binary closed subbase.

Fix a compact metric space $X$ and let $\left\{C_{n}: n \in \omega\right\}$ be a closed base for $X$. We shall construct a sequence $\left\{\mathscr{F}_{n}: n \in \omega\right\}$ of finite families of closed sets such that for each $n \in \omega$,

(1) $\cup \mathscr{F}_{m}=C_{n}$,

(2) $\cup_{m<n} \mathscr{F}_{n}$ is binary.

By (1), $\delta=\cup_{n \in \omega} \mathscr{F}_{n}$ is a subbase for $X$; since $X$ is compact, (2) implies that $\mathcal{S}$ is binary.

We proceed by induction on $n$. Set

$$
\mathscr{F}=\bigcup_{m<n} \mathscr{F}_{m} .
$$

Observe that by induction hypothesis $\mathscr{F}$ is finite and binary. For $p \in C_{n}$, let $\# p=|\{F \in \mathcal{F}: p \in F\}|$ and let

$$
A_{k}=\left\{x \in C_{n}: \# x \geqslant k\right\} \text {. }
$$

Received by the editors February 13, 1978 and, in revised form, May 15, 1978. AMS (MOS) subject classifications (1970). Primary 54D35.

(C) 1979 American Mathematical Society 0002-9939/79/0000-0120/\$01.75 
Note that there is $k_{0}$ such that $A_{k_{0}}=\varnothing$.

We define by downward induction on $k$ a finite set $\mathcal{S}_{k}$ of closed subsets of $X$ such that

(1) $A_{k} \subset \cup \mathcal{G}_{k} \subset C_{n}$;

(2) $\mathcal{S}_{k} \cup \mathcal{F}$ is binary;

(3) $\cup \mathcal{G}_{k}$ is a neighborhood (relative to $C_{n}$ ) of $A_{k}$;

(4) For every $\mathscr{Q} \subset \mathcal{F}$, either $\cap \mathbb{Q} \backslash \cup \mathcal{G}_{k}$ is infinite or $\cap \mathbb{Q}$ $\cap\left(C_{n} \backslash \cup \mathcal{G}_{k}\right)$ is finite.

By (1) and (2), we may take $\mathscr{F}_{n}=\mathcal{G}_{0}$, so it remains only to construct the $\mathcal{G}_{k}$ 's. Take $\mathcal{G}_{k_{0}}=\varnothing$. Fix $k<k_{0}$ and assume $\mathcal{G}_{k+1}$ has been constructed. Set

$$
C=\overline{C_{n} \backslash \bigcup \mathcal{G}_{k+1}} \text {; }
$$

let $\mathcal{H}$ be the set of all intersections with $C$ of intersections of precisely $k$ distinct members of $\mathscr{F}$. By (3), $\mathcal{H}$ is a disjoint collection; also, $\mathcal{H}$ is finite. For each $\mathbb{C} \subset \mathcal{F}$ such that $\cap \mathbb{Q}$ has a limit point in $C \backslash \cup \mathcal{H}$, pick $x_{\mathbb{Q}}$ to be such a limit. Since $\mathscr{F}$ is finite and $X$ is normal, there is a neighborhood $N$ of $\cup \mathcal{H}$ such that $\bar{N} \cap\left\{x_{\mathscr{Q}}: \mathbb{Q} \subset \mathcal{F} \& \cap \mathbb{Q}\right.$ has a limit in $\left.C \backslash \cup \mathcal{H}\right\}=\varnothing$. If $\mathcal{G}_{k}$ is chosen so that $\cup \mathcal{H} \subset \cup \mathcal{G}_{k} \cap C \subset \bar{N}$, (4) will be satisfied. Since $\mathscr{F} \cup \mathcal{G}_{k+1}$ is finite and $X$ is normal, we may pick a finite disjoint cover $\mathscr{U}$ of $\cup \mathcal{H}$ consisting of:

(1) For each infinite $H \in \mathcal{H}$, a neighborhood $U_{H}$ of $H$ such that $\overline{U_{H}}$ meets no members of $\mathscr{F} \cup \mathcal{G}_{k+1}$ that do not meet $H$.

(2) For each finite $H \in \mathcal{H}$ and each $p \in H$, a neighborhood $U_{p}$ of $p$ such that $\bar{U}_{p}$ meets no members of $\mathscr{F} \cup \mathcal{G}_{k+1}$ that do not contain $p$.

Set $\mathcal{G}_{k}^{0}=\{\bar{U} \cap \bar{N} \cap C: \quad U \in \mathcal{Q}\}:$ if $G=\overline{U_{H}} \cap \bar{N} \cap C$ (respectively $G=\bar{U}_{p} \cap \bar{N} \cap C$ ) write $G=G_{H}$ (respectively $G=G_{p}$ ).

If $\mathscr{L} \subset \mathcal{G}_{k}^{0} \cup \mathcal{G}_{k+1}$ and $\mathcal{L}$ is linked, then since $\mathcal{G}_{k}^{0}$ is disjoint, $\mathcal{E}$ contains at most one member $G$ of $\mathcal{G}_{k}^{0}$. If $G=G_{H}$ for some $H \in \mathcal{H}$, pick $p_{\mathfrak{L}} \in H \backslash$ $\cup \mathcal{G}_{k+1}$ in such a way that $p_{\mathscr{T}}=p_{\mathcal{R}}$ only if $\mathfrak{N}=\mathcal{L}$; this is possible since (by (4)) $H \backslash \cup \mathcal{G}_{k+1}$ is infinite. For $G \in \mathcal{G}_{k+1}$ let $G^{\prime}=G \cup\left\{p_{\mathfrak{Q}}: G \in \mathcal{E}\right\}$. Observe that $G^{\prime} \backslash G$ is finite, so $G^{\prime}$ is closed. Set $\mathcal{G}_{k}=\mathcal{G}_{k}^{0} \cup\left\{G^{\prime}: G \in\right.$ $\left.\mathcal{G}_{k+1}\right\}$. It is clear that (1), (3), and (4) hold of $\mathcal{G}_{k}$; it remains only to prove that $\mathcal{G}_{k} \cup \mathcal{F}$ is binary. Let $\mathcal{L} \subset \mathcal{G}_{k} \cup \mathcal{F}$ be linked.

Claim. $\mathcal{L}^{\prime}=\left\{G \in \mathcal{G}_{k+1}: G^{\prime} \in \mathcal{L}\right\}$ is linked. For assume that $G_{0}, G_{1} \in \mathcal{L}^{\prime}$. Since $G_{0}^{\prime} \cap G_{1}^{\prime} \neq \varnothing$ either $G_{0} \cap G_{1} \neq \varnothing$ or there is $p_{\mathbb{E}^{\prime \prime}} \in G_{0}^{\prime} \cap G_{1}^{\prime}$. But this implies that $G_{0}, G_{1} \in \mathcal{L}^{\prime \prime}$ : since $\mathcal{L}^{\prime \prime}$ is linked, $G_{0} \cap G_{1} \neq \varnothing$.

There are three cases to consider.

Case 1. $\mathcal{L}=\left\{F_{0}, \ldots, F_{n}\right\} \cup\left\{G_{0}^{\prime}, \ldots, G_{m}^{\prime}\right\}$ for some $F_{0}, \ldots, F_{n} \in \mathscr{F}$.

Then $\cap \mathcal{L} F_{0} \cap \cdots \cap F_{n} \cap \mathcal{L}^{\prime}$ which is nonempty by induction hypothesis.

Case 2. $\mathcal{L}=\left\{G_{p}\right\} \cup\left\{F_{0}, \ldots, F_{n}\right\} \cup\left\{G_{0}^{\prime}, \ldots, G_{m}^{\prime}\right\}$.

Then by construction (of $G_{p}$ ), $p \in \cap \mathcal{L}$.

Case 3. $\mathcal{L}=\left\{G_{H}\right\} \cup\left\{F_{0}, \ldots, F_{n}\right\} \cup\left\{G_{0}^{\prime}, \ldots, G_{m}^{\prime}\right\}$. 
By choice of $G_{H}$, each $F_{i}$ contains $H$; in particular, $p_{\mathfrak{L}^{\prime}} \in F_{0} \cap \cdots \cap F_{n}$. Then $p_{\mathfrak{L}^{\prime}} \in \cap \mathcal{L}$.

\section{REFERENCES}

1. M. G. Bell, Not all compact Hausdorff spaces are supercompact, General Topology and Appl. 8 (1978), 151-155.

2. M. G. Bell and J. van Mill, The compactness number of a compact topological space, Fund. Math. (to appear).

3. E. K. van Douwen and J. van Mill, Supercompact spaces, General Topology and Appl. (to appear).

4. E. K. van Douwen, Special bases for compact metric spaces, Fund. Math. (to appear).

5. J. De Groot, Supercompactness and superextensions, Contributions to Extension Theory of Topological Structures (Sympos. Berlin, 1967), Deutscher Verlag Wiss., Berlin, 1969, pp. 89-90.

6. C. F. Mills, Compact groups are supercompact (to appear).

7. M. Strok and A. Szymański, Compact metric spaces have binary bases, Fund. Math. 89 (1975), 81-91.

Department of Mathematics, University of Wisconsin, Madison, Wisconsin 53706 